\title{
MORPHOLOGICAL OBSERVATIONS ON WOOL FABRICS TREATED WITH POTASSIUM CYANIDE IN MIXTURES OF WATER AND ORGANIC SOLVENTS
}

\author{
Fumi Masuko*1, Ken-ichi Furuhata ${ }^{* 2}$, and Munenori Sakamoto ${ }^{* 2}$ \\ *1 Department of Clothing. Japan Women's University, Mejirodai, Bunkyo-ku, Tokyo \\ 112 Japan \\ *2 Department of Organic and Polymeric Materials, Faculty of Engineering, Tokyo \\ Institute of Technology, O-okayama, Meguro-ku, Tokyo, 152 Japan
}

\begin{abstract}
Merino wool fabrics were treated with dilute $\mathrm{KCN}$ in water/organic solvent mixtures, and resulting morphological changes were studied by scanning electron microscopy. Longitudinal striations were differentially formed on the orthocortex side of the fiber surface.
\end{abstract}

Wool properties depend much on the content and the distribution of cystine linkages which form interand intramolecular crosslinks. Cystine linkages in wool are cleaved via $\mathrm{S}_{\mathrm{N}} 2$ mechanism when attacked by cyanide ions, and the resulting cystine and $S$ cyanocysteine residues react to give lanthionine residues $[1-3]$. The cysteine residue and the $S$ cyanocysteine residue that combine to form one particular lanthionine residue are not necessarily those derived from the same cystine residue. The crosslinked structure of wool could be changed by the reac. tion, depending on the reaction conditions. It was shown previously $[4,5]$ that wool fibers became more susceptible to swelling in conc. formic acid after treated with aq. $\mathrm{KCN}$ although the total amount of crosslinkable cystine and lanthionine residues was changed little by the treatment.

The preceding paper $(5)$ reported that significant changes in the structure of fabrics, yarns, and fibers were observed when wool fabrics were treated with $\mathrm{KCN}$ in various mixtures of water and water-miscible organic solvents. This paper briefly describes further study on the wool fabrics treated with KCN in mix. tures of water with acetone or 2-propanol.

Merino wool serge fabrics $\left(304 \mathrm{~g} / \mathrm{m}^{2}\right)$, Soxhlet-ex. tracted with ethanol and air-dried, were used in this study. Fabrics were treated with $0.08 \mathrm{M} \mathrm{KCN}$ in wa ter or in $3: 1(\mathrm{v} / \mathrm{v})$ mixtures of water with acetone or with 2-propanol at $80^{\circ} \mathrm{C}$ for $30 \mathrm{~min}$, rinsed with wa- ter, then, with acetone, and air-dried. Scanning elec tron microscopic (SEM) observation was made on Hitachi scanning electron microscopes, S-430 and S800 , with accelerating voltages of 5 and $15 \mathrm{kV}$. Wool samples were coated with $\mathrm{Au}$ and $\mathrm{Pd}$ by ion spattering.

One of the apparent changes caused by the $\mathrm{KCN}$ treatment was shrinkage of the fabric. Lengthwise and widthwise shrinkages (\%, warp + filling) were $11.2,13.7$, and $17.5 \%$ for the treatments in water aq. acetone, and aq. 2-propanol, respectively. The shrinking took place during deswelling; the fabric started to shrink not in $\mathrm{KCN}$ solutions but in water for washing and continued to shrink during airdrying.

Another apparent change by the $\mathrm{KCN}$ treatment was, as reported previously [5], formation of pinholes at the intersections of the warp and filling yarns of the fabric when the reaction was carried out in mix tures of water with organic solvents. The diameters of the pinholes determined in this work by SEM were about $50-60$ and $100 \mu \mathrm{m}$ for the fabrics treated in aq. acetone and in aq. 2-propanol, respectively.

Fig. 1 compares the untreated fabric and the fabric treated in aq. acetone. The fibers in the yarns in the untreated fabric are more loosely arranged and less oriented while the fibers in the $\mathrm{KCN}$-treated yarns are very densely arranged and more oriented to the yarn direction. 


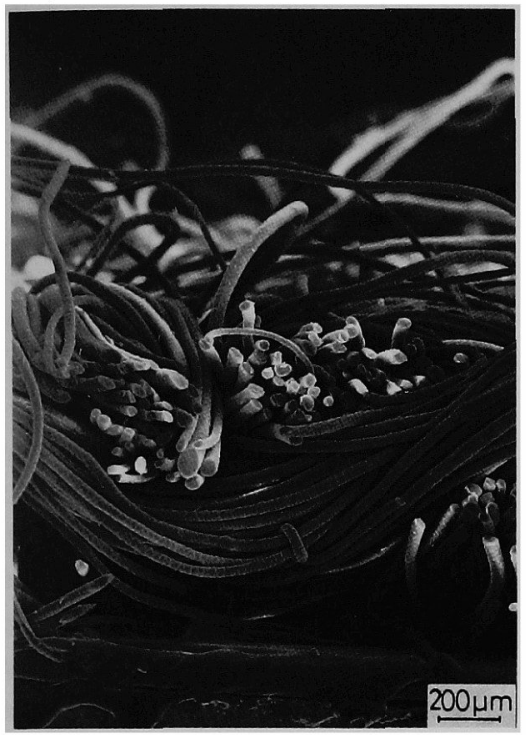

(a)

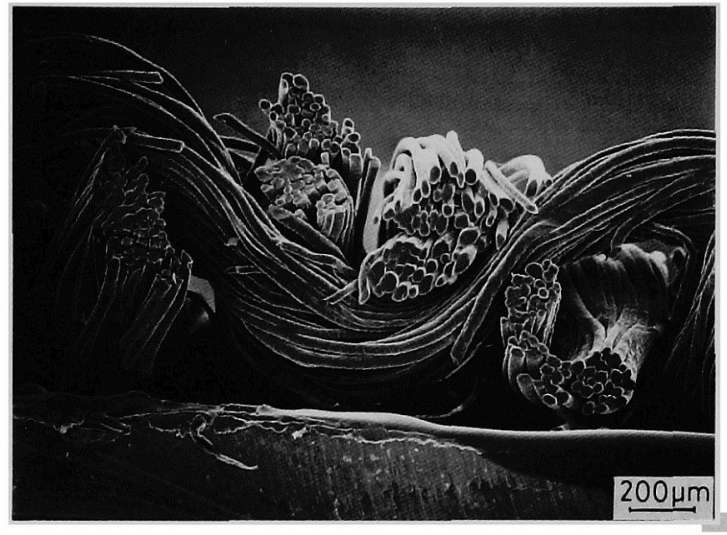

(b)

Fig. 1. SEM photographs of the warp cross sections of Merino wool serge fabrics: (a) untreated. (b) treated with 0.08 $\mathrm{M} \mathrm{KCN}$ in a $3: 1(\mathrm{v} / \mathrm{v})$ mixture of water and acetone at $80^{\circ} \mathrm{C}$ for $30 \mathrm{~min}$.

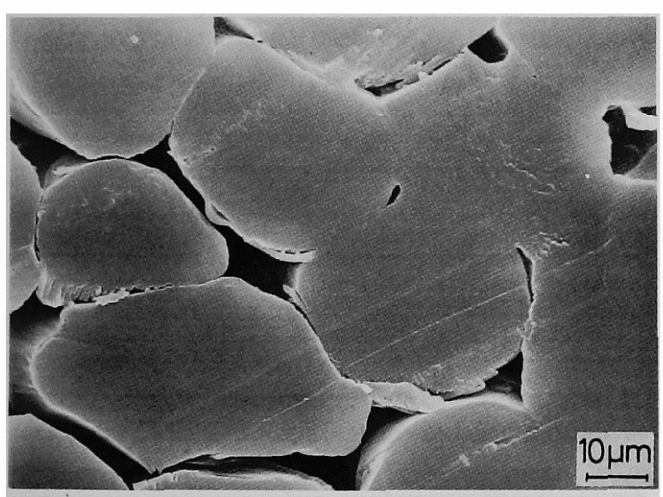

(a)

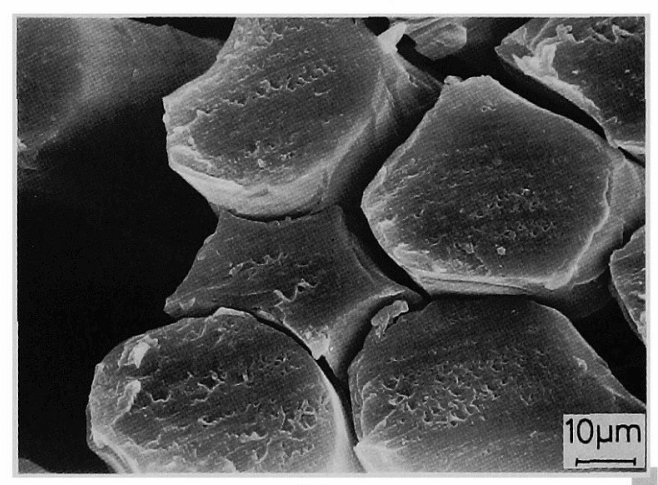

(b)

Fig. 2. SEM photographs of the croos sections of warp yarns of the wool fabrics treated with $0.08 \mathrm{M} \mathrm{KCN}$ in $3: 1$ (v/v) mixtures of water and 2-propanol under (a) unrestricted conditions and (b) restricted conditions where warp length was kept to the original throughout the treatment, washing, and air drying.

Fig. 2(a) shows that the cross section of a yarn of the fabric treated with $\mathrm{KCN}$ in aq. 2-propanol. This higher magnification picture clearly shows that the treated yarn is very densely filled with fibers. The fiber cross sections are slightly deformed and the fibers touch each other at many parts. Furthermore, the fiber contours often disappear and the fibers look adhered closely to one another.

Non-keratinous proteins and degraded products are known to be extracted when wool fibers are treated in alkaline solutions [6]. Such extracts or wool glues may deposit on the surface of fibers and glue fibers. Fig. 3 shows that wool glues deposit on the surface of fibers and fill the space between fibers when a wool fabric is treated with aqueous $\mathrm{KCN}$ and insufficiently washed only with water. For completely washed fabrics, no such glues were found. Therefore, the fiberto-fiber adhesion, such as in Fig. 2(a), is not related 


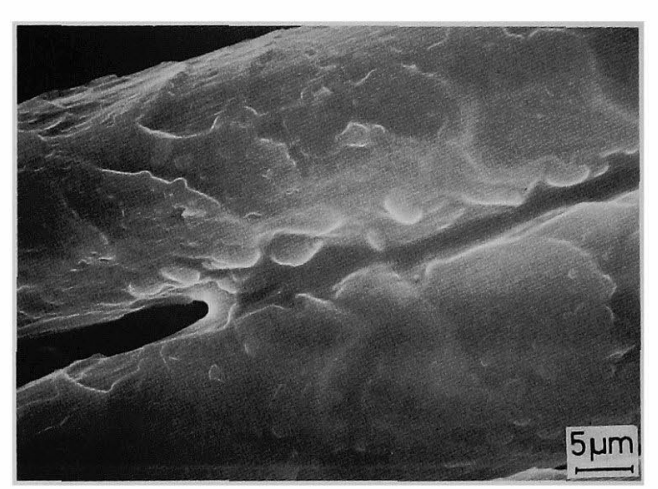

Fig. 3. SEM photograph of fibers of the wool fabric treated with $0.08 \mathrm{M}$ aqueous $\mathrm{KCN}$ at $80^{\circ} \mathrm{C}$ for $30 \mathrm{~min}$ which was washed only with water insufficiently.

to such glues.

Wool fabric was treated with $\mathrm{KCN}$ in aq. acetone with the warp length being fixed at the original length on a metal frame during the treatment, washing, and air drying. Fig. 2(b) shows that the deformation of the fiber cross section is very significant; many fibers show pentagonal cross sections. No such characteristic deformation has been reported in the literature. Some of the fibers (not seen in Fig. 2(b)) adhere to each other under unrestrictive conditions.

The change in the yarn and fiber structure shown above probably occurs in the following manner. Individual fibers are swollen in the reaction medium. When the swollen fabric is placed into water, the fibers will shrink eventually. ${ }^{\# 1}$ Naturally, the fibers in

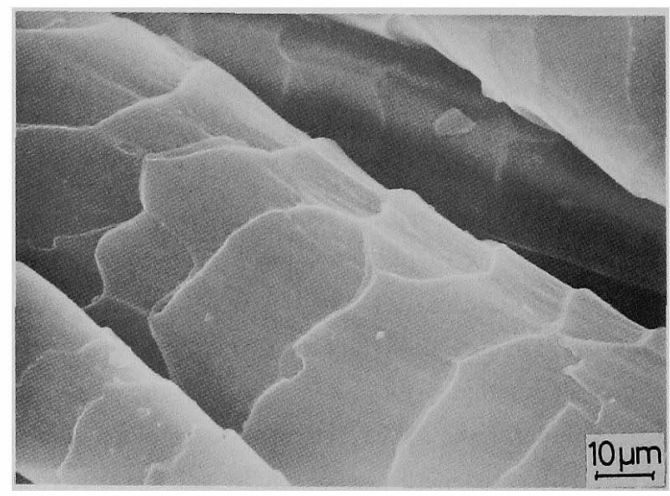

(a) the outer shells of the yarns shrink first and the shrunk fibers will press the fibers in the cores of the yarns. This pressure makes the yarn structure denser and the swollen and mechanically weak fibers are deformed and they adhere to each other. The tension developed in the swollen yarns, when placed in washing water with the length of the fabric forced to be unchanged, affected much the shape of the cross section of the deswollen fibers.

All the changes in the fabric, yarn, and fiber structures were the most significant when fabrics were treated in aq. 2-propanol and the least when treated in water. Aq. 2-propanol is effective to cleave both hydrogen bonds and hydrophobic bonds in wool. It is expected that wool fibers are the most swollen in aq. 2-propanol among the solvents used in this study and this can explain why the deformation was the most significant for the treatment in that medium.

In the preceding paper [5], deep longitudinal striations were reported to be formed on the fiber surface by the treatment with $\mathrm{KCN}$ in mixtures of water with organic solvents. Such deep striations are occasionally reported in the literature $[8,9]$. Extensive SEM reobservation on the $\mathrm{KCN}$-treated samples in this

\footnotetext{
\#1 It is possible that the swollen fibers further swell rapidly and extensively when placed in water owing to the osmotic pressure developed. However, salt ions in swollen wool fibers come out gradually from the fibers and shrinking or deswelling will follow. Such spontaneous swelling and deswelling behavior was observed recently when wool fibers treated with a reducing agent were placed in water [7].
}

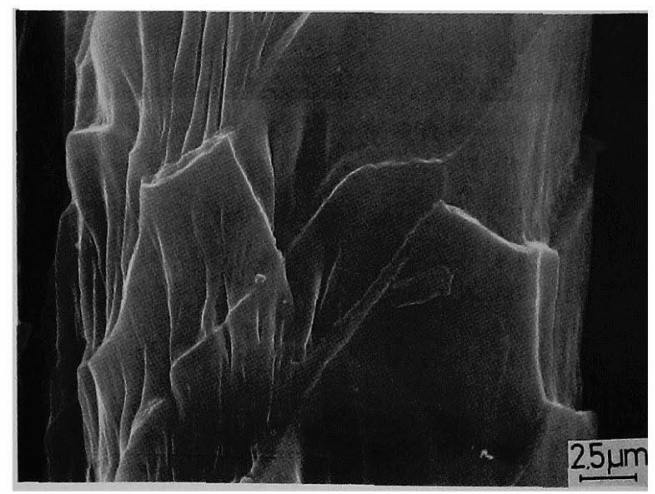

(b)

Fig. 4. SEM photographs of (a) untreated wool fiber and (b) a fiber of the wool fabric treated with $0.08 \mathrm{M} \mathrm{KCN}$ in a 3 $: 1(\mathrm{v} / \mathrm{v})$ mix,ture of water and 2 -propanol at $80^{\circ} \mathrm{C}$ for $30 \mathrm{~min}$. 


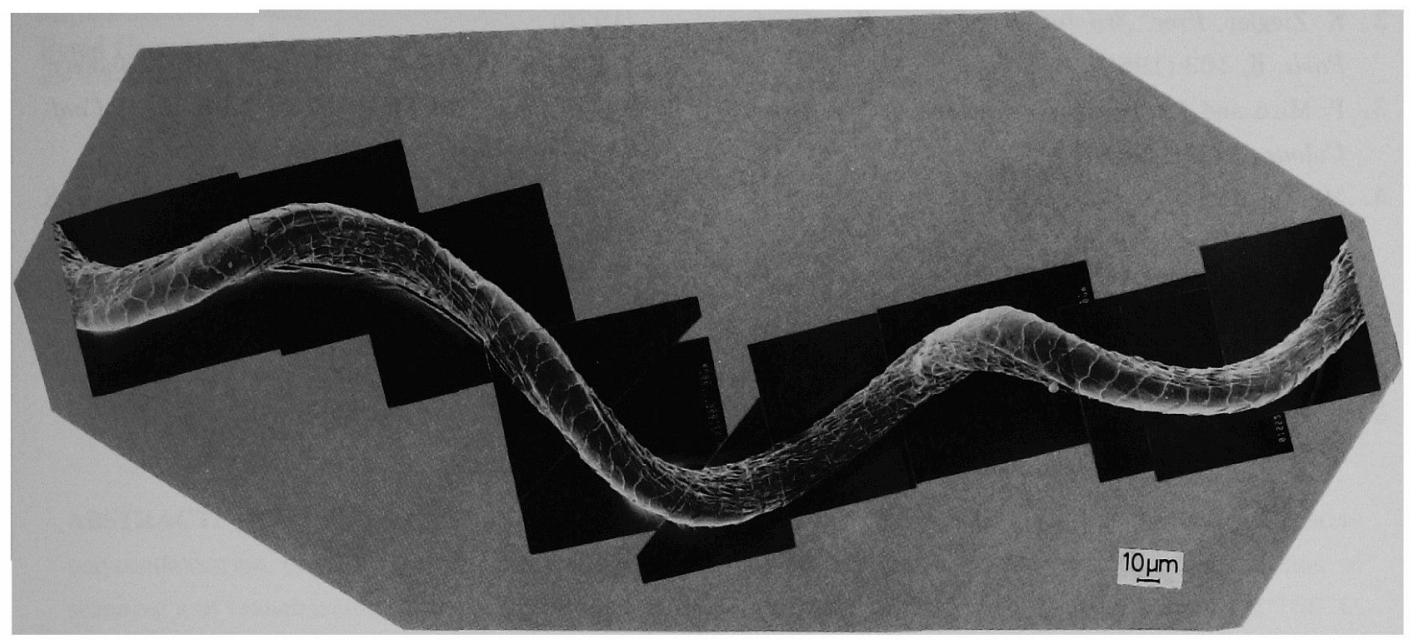

Fig. 5. A synthesized SEM photograph of a single Merino fiber treated with $0.08 \mathrm{M} \mathrm{KCN}$ in a $3: 1(\mathrm{v} / \mathrm{v})$ mixture of water and 2-propanol at $80^{\circ} \mathrm{C}$ for $30 \mathrm{~min}$.

study led to an idea that the striations were formed differentially because of the well-known bilateral structure [10] of wool fibers. Fig. 4 compares the surface of an untreated fiber and that of a fiber in the fabric treated with $\mathrm{KCN}$ in aq. acetone. The latter is a typical case showing that longitudinal striations are seen on one side of the fiber surface. Longitudinal striations were also formed differentially for the samples treated with aq. KCN. The striations were finer and shallower in this case.

Wool fibers consist mainly of two types of cortical cells and these cells comprise the orthocortex and the paracortex. The two types of cortices in Merino wool fibers are located bilaterally; the orthocortex is always located on the outside and the paracortex always on the inside of the natural wool crimp wave [10].

The SEM observation of the KCN-treated wool fibers suggests that the longitudinal striations are preferentially formed on one particular side of the two cortices. The orthocortex is reported to swell laterally in dilute aqueous alkali more than the paracortex, therefore, the striations are expected to be formed on the orthocortex side.

A bulk of single Merino fibers were treated with $0.08 \mathrm{M} \mathrm{KCN}$ in aq. 2-propanol at $80^{\circ} \mathrm{C}$ for $30 \mathrm{~min}$ and a single fiber taken from the bulk was observed by SEM. Fig. 5 clearly shows that the striations are formed always on the inside of the crimp wave.

In order to determine on which cortex the striations were formed, the KCN-treated fibers were dyed with a basic dye, Janus Green. Microscopic observation of the stained fiber showed that the heavily stained orthocortex was located on the inside of the crimp wave and not on the outer side as was for the untreated fiber. Apparently the crimp wave structure was reversed by the $\mathrm{KCN}$ treatment: the inner side of the crimp comprising the orthocortex is transformed to the outer side and the outer side comprising the paracortex to the inner side. Horio and Kondo [10] reported that in a dilute sodium hydroxide (up to $5 \%$ ), the orthocortex preferentially swelled laterally and shrank longitudinally, and this led to the reversed structure of the crimp. Since the $\mathrm{KCN}$ solution is slightly alkaline, the formation of a similar reversed crimp structure by the treatment is very likely to occur. These results lead to a conclusion that the formation of the longitudinal striations is caused by lateral swelling of the orthocortex followed by shrinking during washing and drying.

More details of the present study will be reported later.

\section{REFERENCES}

1. K. Earland and D. J. Raven, J. Am. Chem. Soc., 80, 3430 (1958) 
2. K. Ziegler, Proc. 3rd Int. Wool Text. Res. Conf., Paris, II, 403 (1965)

3. P. Miró and J. J. Garcia-Dominguez, J. Soc. Dyers Colour., 83, 91 (1967)

4. M. Sakamoto, K. Kajiyama, M. Iwata, and H. Tonami, Proc. 5th Int. Wool Text. Res. Conf., Aachen, III, 125 (1975)

5. K. Kajiyama, M. Iwata, M. Sakamoto, and H. Tonami, Sen'i Gakkaishi, 34, T.259 (1978)

6. H. Baumann and L. Möchel, Textilvered., 11, 238
(1976)

7. J. Nöthen, V. Bölert, H. Höcker, and G. Blankenburg, Proc. 8th Int. Wool Text. Res. Conf., Christchurch, I, 307 (1990)

8. A. Hepworth, J. Sikorski, D. J. Tucker, and C. S. Whewell, J. Text. Inst., 60, 513 (1969)

9. M. E. Campbell and H. Baumann, Text. Res. J., 51, 52 (1981)

10. M. Horio and T. Kondo, Text. Res. J., 23, 373 (1953) 